\title{
Infrared Attenuation of Thallium Bromo-lodide Fibers
}

\section{Beryl Magilavy and John Goebel}

February 1986 


\section{Infrared Attenuation of Thallium Bromo-lodide Fibers}

Beryl Magılavy, Informatics, Inc., Palo Alto, California

John Goebel, Ames Research Center, Moffett Field, Calıfornıa

February 1986

\section{N/S}

Natıonal Aeronautics and

Space Admınıstratıon

Ames Research Center

Moffett Field, Calıfornia 94035 


\title{
INFRARED ATTENUATION OF THALLIUM BROMO-IODIDE FIBERS
}

\author{
Beryl Magilavy* and John Goebel \\ Ames Research Center
}

\section{SUMMARY}

Analysis of attenuation measurements in the near infrared of an unclad fiber of Thallium Bromo-Iodide ( $\mathrm{Th}(\mathrm{Br}, \mathrm{I})$ ), a polycrystalline thallium halide, is presented. The first section of the paper is devoted to a general overview of the properties of fiber optics. Two groups of attenuation measurements, for the region 1.2 to $3.4 \mu \mathrm{m}$ and for 3 to $11 \mu \mathrm{m}$, respectively, are presented, analyzed, and compared with those of two other groups of researchers. Materials details of $\mathrm{Th}(\mathrm{Br}, \mathrm{I})$ are included as appendix A.

\section{INTRODUCTION}

In the past 5 years there has been increasing interest in the optical community in developing materials to transmit light at wavelengths longer than the 1.3- to 1.5- $\mu \mathrm{m}$ band currently in use. Metal halides, thallium halidßs such as $\mathrm{Th}(\mathrm{Br}, \mathrm{I})$ (commonly and henceforth referred to as KRS-5), and fluoride glasses have shown the most promise for the 10- $\mathrm{m}$ region. Theoretical transmission curves for several materials are given in figures 1-3 (refs. 1-3). Few of these materials are yet avallable commercially, since their performance is still being examined and improvements are being made in their fabrication. The work described in this paper is part of an ongoing project which is aimed at identifying and characterizing new materials for use as infrared fibers.

In this paper experimental work to measure the attenuation of KRS-5 fibers is described. The first section is a brief history and description of the physics of optical fibers. Only those topics necessary for understanding this work will be covered here: total internal reflection, numerical aperture, mode structure, and attenuation.

The second section of the paper presents measurements made in 1982 by researchers at NASA Ames Research Center of attenuation at 1.2 and $3.4 \mu \mathrm{m}$ and measurements of attenuation at 3 to $11 \mu \mathrm{m}$ made in 1985. These data are compared w1th published measurements of KRS-5 attenuation by two other groups of researchers. Appendices $A$ and $B$ provide a detalled presentation of the data and summarize the materials properties of KRS- 5 .

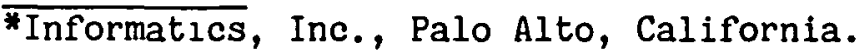




\section{HISTORY}

The technique of guiding light through tubes or fibers employs the process of total internal reflection at a dielectric interface, a phenomenon first demonstrated by John Tyndall in 1854 at the Royal Institute in London. He "permitted water to flow from a tube, the light on reaching the limiting surface of air and water was totally reflected and seemed to be washed downward by the descending liquid. . ." (ref. 4).

Patents for the process of image transfer were issued in the United States and the United Kingdom in the late 1920s. Since uncoated fibers were used in the early work, efficiency was low. In the 1950s, van Heel in Holland began coating his flbers with a solid cladding with a refractive index lower than that of the core. Kapany, who published a major paper on fiber efficiency in England, simultaneously with van Heel, did most of the early development work, along with the American Optical Company. Various technical advances (including the introduction of a second, absorbing cladding to minimize stray light) occurred in the next decade, but efficiency was considered unacceptably low, with attenuation of about $1000 \mathrm{~dB} / \mathrm{km}$. When one considers the loss relationship

$$
\text { loss }(\text { in } d B)=10 \log _{10}\left(\text { Power }_{\text {in }} / \text { Power }_{\text {out }}\right)
$$

then over $1 \mathrm{~km}$, an attenuation of $1000 \mathrm{~dB} / \mathrm{km}$ would amount to an output to input ratio of $10^{-100}$. In 1970 , low losses of $20 \mathrm{~dB} / \mathrm{km}$ in fused-silica glass were announced, and reported materials research during the next decade concentrated almost solely on this material. To date, no other materials have been supplied commercially. The KRS -5 fibers used in this work were provided by the optics research division of Harshaw Chemical Company.

Silica has optimum transmission in the optical region of 1.3 to $1.5 \mu \mathrm{m}$, and current fiber-transmissions applications are almost exclusively limited to this range. There is an intrinsic limit to the efficiency of any particular fiber. The limit for silica fibers has been calculated at $0.2 \mathrm{~dB} / \mathrm{km}$ (loss over $1 \mathrm{~km}$ of about 5\% (ref. 5)). Present commercial fibers achleve close to this level of efficiency. Impetus to develop new materials was provided by the desire to reduce the absolute level of attenuation and to enable transmissions at other wavelengths.

\section{ELEMENTARY FIBER THEORY}

Many texts are available on elementary fiber theory; two good ones are W. B. Allan's Fibre Optics, Theory and Practice (ref. 4) and N. S. Kapany's Fiber Optics (ref. 6). 
The diameter of the fibers used in this study is $381 \mu \mathrm{m}$, out of a typical fiber range of 125 to $400 \mu \mathrm{m}$. (For comparison, a human hair has a diameter of about $50 \mu \mathrm{m}$.$) Even the longer wavelengths used in our measurements can be considered$ small by comparison. The wave nature of the radiation can be ignored, and the propagation can be described by the simpler laws of geometrical optics.

The phenomenon of the bending of light at the interface of two transparent media was first noted by the ancient Greeks and quantified in 1621 by Snell, whose construction required the ratios of the cosecants of the incident and reflected angles to be constant. The current sine form of the relation was first introduced by Descartes (and called Descartes' Law in France). The equation

$$
\mathrm{n}_{1} \sin \theta_{1}=\mathrm{n}_{2} \sin \theta_{2}
$$

is known elsewhere as Snell's Law, where $\mathrm{n}$ is the index of refraction in the incident or transmitting medium. The subscript convention we will use is that $n_{1}$ is the higher fiber index of refraction, which corresponds to the core index in fibers; we will use $\mathrm{N}_{0}$ to represent the index of the surrounding medium (e.g., alr), and we will use $\theta$ as the angle of deviation from the normal. When $\theta_{2}>90^{\circ}$, no light $w 11 l$ be transmitted, and total internal reflection will result. The critical angle $\theta_{c}$, at which total internal reflection occurs, is $\sin ^{-1}\left(n_{2} / n_{1}\right)$ (fig. 4).

\section{Numerical Aperture}

Knowledge of the numerical aperture is essential in determining how much of the output of a power source actually enters the fiber. Figure 5 illustrates that since only rays hitting the fiber wall at angles from the normal that are less than $\theta_{c}$ will be internally reflected, a cone exists in the space adjacent to the fiber end from outside of which light will not be trapped in the fiber. This index of the light-gathering power of the fiber is known as the numerical aperture and is calculated for most materials

$$
N A=\sqrt{n_{1}^{2}-n_{2}^{2}}=n_{0} \sin \theta_{A}
$$

where:

$$
\begin{aligned}
& n_{1}=\text { core index } \\
& n_{2}=\text { cladding index } \\
& n_{0}=\text { index of surrounding medium; (for this case, } n_{0}=n_{a i r}=1 \text { ) } \\
& \theta_{A}=\text { angle of the incident ray from the fiber end normal } \\
& N A=\text { numerical aperture }
\end{aligned}
$$

For an unclad fiber, $n_{2}=n_{0}=1$ and this expression will give the aperture if the core index of refraction is $<\sqrt{2}$. For fibers with an index of $\sqrt{2}$ or greater, all the light impinging on the fiber end is totally reflected. Figure 6 illustrates this phenomenon. Assume that a ray of light enters the fiber end at nearly $90^{\circ}$ from 
the end normal. By Snell's law, the light will be transmitted into the fiber at an angle of almost $25^{\circ}$. The ray reaches the fiber wall at an angle of about $65^{\circ}$. The critical angle at the fiber wall at which $n_{1} \sin \theta_{1}=1$ is $25^{\circ}$ (as is clear if you retrace the ray's path). Therefore, since any ray reaching the wall surface is at an angle from the wall normal greater than $25^{\circ}$, no light will escape the fiber.

The numerical aperture is wavelength-dependent since the index of refraction is a function of the wavelength.

\section{Mode Structure}

Depending on the index of refraction and diameter of a fiber, l1ght waves will be guided in either single or multimode forms. A mode is the number of reflections a ray encounters in its journey down a fiber. The total number of modes depends on the $V$ number, a number which characterizes the fiber in terms of its modal structure; in equation form

$$
\mathrm{V}=\mathrm{n} k \mathrm{r} \sqrt{2 \Delta}
$$

where

$$
\begin{aligned}
& \mathrm{n}=\text { core index } \\
& \mathrm{k}=\text { wavenumber (related to the wavelength as } \mathrm{k}=2 \pi / \lambda \text { ) } \\
& r=\text { core radius } \\
& \Delta=\left(n^{2}-n_{1}^{2}\right) / 2 n^{2} \quad \text { where } \\
& \mathrm{n}=\text { core index } \\
& \mathrm{n}_{1}=\text { cladding index }
\end{aligned}
$$

The convention is to consider $V<2.4$ a single-mode fiber; the desired cut-off wavelength sets a limit to the possible fiber diameter, usually about one-f $1 \mathrm{fth}$ that of multimode fibers. The primary advantage of single-mode propagation is that it will carry relatively broadband signals. Multimode fibers, on the other hand, have a limited bandwidth past which dispersion becomes unacceptable. With a $V$ number of 613 at $10 \mu \mathrm{m}$, the large KRS-5 fibers used here would carry multimode signals.

\section{Attenuation}

Attenuation is the removal of power from the light ray in the fiber, exclusive of losses from outside causes such as reflections on the end surfaces of the fiber or detector. Attenuation consists of absorption and scattering which may arise from intrinsic causes (l.e., those fundamental to the structure of the material) or extrinsic causes (i.e., those potentially removable by improvements in fiber manufacture or measurement technique). The wavelength dependence of various mechanisms is illustrated in figure 7 (ref. 7 ).

The total attenuation coefficient $\alpha_{t}$ of light propagating in an optical fiber can be written in the form 
where

$$
a_{t}(\lambda)=A \lambda^{-4}+B \lambda^{-2}+a_{a}(\lambda)+c
$$

$\lambda=$ wavelength

$\alpha_{t}=$ total attenuation

$\alpha_{a}=$ absorption coefficient

$c=$ constant

The $\lambda^{-4}$ component, attributed to material scattering, is the dominant element in fused-silica fibers. The $\lambda^{-2}$ term arises from bulk scattering out of the incident beam, plus surface scattering. The absorption coefficient $\alpha_{a}$ varies with wavelength, and the constant term is due to the presence of impurities. Each of these elements will be discussed in turn.

Materials scattering: $\lambda^{-4}$ dependence- In 1980, Mie found that the solution for the diffraction of a plane electromagnetic wave by a homogeneous sphere of radius $<<\lambda$ describes a wave having amplitude in inverse square to the wavelength. Since intensity is proportional to the square of the amplitude, the intensity of light scattered off a sphere varies as $\lambda^{-4}$ (should the radius not be $<<\lambda$, this relation will no longer hold) (ref. 8).

Though materials scattering falls off rapidly with increasing wavelength, it is significant in the range below $3 \mu \mathrm{m}$ (figs. 1 and 7 ). Inside a fiber, materials scattering occurs because of microscopic density fluctuations in the medium. Each area of uniform density that is different from its surroundings can be considered roughly equivalent to Mie's homogeneous sphere. The direction of the light ray can be changed with no change in frequency (Rayleigh scattering) or with a decrease in frequency at the same time (Raman scattering). A $\lambda^{-4}$ relation between wavelength and attenuation level also results from the interaction of the incident light with thermal fluctuations in the lattice structure of the medium (Brillouin scattering). Substantial reduction of losses from this source can be achieved from operating in the range above $3 \mu \mathrm{m}$ where Brillouin scattering becomes negligible.

The primary fiber materials are glasses, crystals, and polycrystalline materials. The causes of materials scattering are different in each.

Glasses (scattering caused by macroscopic disorder): In the case of glasses, the amount of nonuniformity in the dielectric constant of the material depends primarily on its fictive (characteristic solidifying) temperature. Pinnow et al. (ref. 9) found the scattering coefficient to be

$$
a_{\text {scatt }}=\lambda^{-4} n^{8} p^{2} B T
$$

where

$$
\begin{aligned}
& n=\text { index of refraction } \\
& p=\text { average photoelastic constant } \\
& \beta=\text { isothermal compressibility } \\
& T=\text { glass fictive temperature }
\end{aligned}
$$


When the fictive temperature is low, then the scattering losses from this cause are low. The transition temperature of fused silica is $1700 \mathrm{~K}$, which is relatively high. Silica has an advantage, however, in being a single-component glass; the statistically random distribution of the polarizable components of multicomponent glass adds to its scatter loss.

Crystals (scattering caused by defects): A perfect crystal would show no absorption in its optical window because it would have no disorder in its structure. Real crystals, however, suffer defects such as vacancy clusters (where cations or anions, or both, are missing from the lattice) and equilibrium-point defects (where an atom from the pure crystal is replaced in the crystal structure by one with a different number of valence electrons), which give rise to microscopic fluctuations in the dielectric constant. Crystals have not been favored for development as fiber optical materials because of the difficulties in drawing them into fibers and because of their relative inflexibllity.

Polycrystalline fibers (scattering caused by structure): A polycrystalline fiber is one made of fused anisotropic crystals. Inherent in these fibers are not only the crystal defects just discussed, but also scattering centers associated with each grain boundary. An electron microscope photograph of the fiber used in these experiments is shown in figure 8 . The individual crystals in this fiber are large compared to some of other manufacture, such as the KRS-5 fiber illustrated in figure 9 (ref. 10). The smaller crystal structure increases disorder, and hence attenuation, but makes the fiber less likely to shatter when it is handled or bent.

Surface scattering: $\lambda^{-2}$ dependence- The work of Gans (ref. 8 ) has shown that scattering with $\lambda^{-2}$-wavelength dependence results from the scattering of waves at the boundary of an ellipsoid (where the ellipsold describes a volume in which the index of refraction is uniform and is different from its surroundings). Scattered power is given by

$$
P_{S C}=\int d \Omega J(\Omega)
$$

where

$$
\begin{aligned}
P_{S C} & =\text { scattered power } \\
J(\Omega) & =\text { radiant intensity }
\end{aligned}
$$

At large sites the amplitude of the diffracted wave is largely confined to the diffraction angle $\theta_{\mathrm{d}} \propto \lambda / 1_{\mathrm{tr}}$, where $l_{\mathrm{tr}}$ is the transverse dimension. Since intensity, $J(\Omega)$, is proportional to the square of the amplitude, $J(\Omega)$ can be considered large only over a solid angle

$$
\Delta \Omega_{S C} \approx \theta_{d}^{2} \approx \lambda^{2}
$$


with

$$
\begin{gathered}
J\left(\Omega_{s c}\right) \propto \lambda^{-4} \\
P_{s c}=J\left(\Omega_{s c}\right) \Delta \Omega_{s c} \propto \lambda^{-4} \lambda^{2}=\lambda^{-2}
\end{gathered}
$$

Harrington (ref. 11) has shown that this type of scattering may be the result of surface irregularities and residual strains in the fiber. Both flaws are introduced during manufacture, and are most severe in soft material of polycrystalline structure such as KRS-5. Figure 10 shows a surface scratch in our fiber, probably caused by dust particles on the surface of the diamond die through which the fiber was extruded. The soft edges of the striations visible at this magnification result from the liquidity of the heated fiber material.

Absorption: $\lambda$ dependence- Absorption that is $\lambda$ dependent is caused by electronic transition absorption, infrared absorption, and impurities absorption.

Electronic transition: Defining the short-wavelength side of the optical window, ultraviolet (UV) absorption takes place when bound electrons in the material oscillate at resonant frequencies $\omega_{0}$ with the impinging radiation. The relationship between the index of refraction and the frequency of incident light is given by

where

$$
n^{2}(\omega)=1+N q_{e}^{2} / \varepsilon m_{e} \sum_{i}\left[f_{i} /\left(\omega_{0}^{2}-\omega^{2}+i Y_{i} \omega\right)\right]
$$

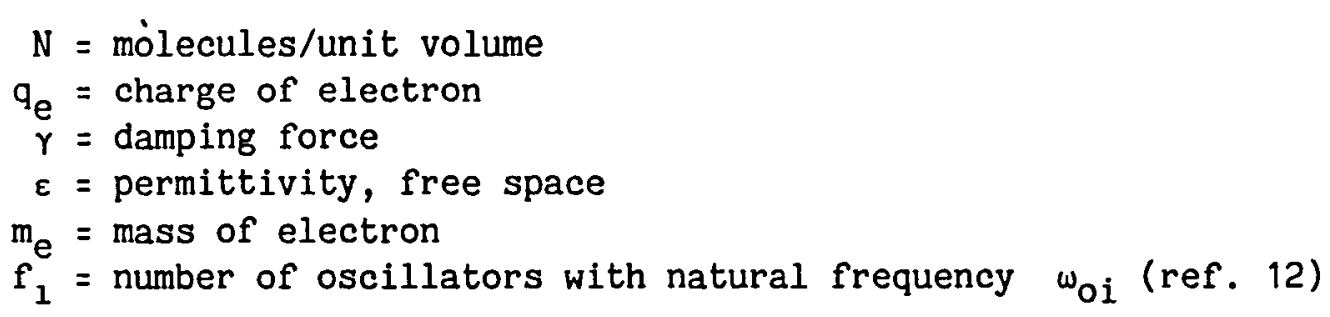

When $\omega=\omega_{0}$, the expression in the summation is dominated by the imaginary damping term. The index of refraction becomes imaginary and absorption results. Associated with this phenomenon is an intrinsic absorption loss in chemically pure materials in what before 1973 was considered to be the completely transparent region below the energy level at which UV absorption takes place. The mechanism for this loss is the local, field-induced broadening of the resonant energy levels to include energles slightly below the normal resonances just described. This absorption is known as the UV or Urbach tall, and falls as $c_{1} \exp \left(c_{2} / \lambda\right)\left(c_{1}\right.$ through $c_{4}$ here and in the following paragraph are experimentally deduced constants.)

Infared edge: On the long-wavelength side of the optical window, infrared absorption occurs, caused by phonon interaction in the lattice structure of the fiber material. Impinging radiation in this range causes vibration of the intermolecular bonds of the fiber and energy is dissipated by frictional losses. These 
edge losses are slightly temperature-dependent. The IR edge exhibits a "tail" into the optical window with a profile of $C_{3} \exp \left(-C_{4} / \lambda\right)$. Goodman (ref. 13) suggests that this absorption tail may be a strain-induced broadening of the IR absorption bands in the related crystalline material.

Impurities absorption: wavelength-independent- The foregoing discussion assumes pure materials, a condition which has been virtually achieved in the fabrication of fused-silica fibers, which have been in production for a decade. The presence of impurities in experimental materials causes characteristics absorption bands at the resonant vibration frequencies of the impurity molecules. In the attenuation data presented in figure 15, for example, the peak which appears at about $9 \mu \mathrm{m}$ in several of the test results may indicate the presence in the fiber of $\mathrm{CO}_{2}$, diffused in from the atmosphere, which has an absorption band at $9.4 \mu \mathrm{m}$. These extrinsic losses can be avoided by advances in fiber manufacture and improvements in storage and cladding, but, as Lines (ref. 14) notes in the Journal of Applied Physics,

The practical difficulties in assessing the low loss potential of the relative materials centers on the fact that material refinement has not yet reached the state for which intrinsic absorption and scattering mechanisms (which would dominate the limiting attenuation in an ideal sample) can be measured. In particular, current absorption measurements in the optic window succeed primarily only in characterizing the imperfections present.

Attenuation coefficient- The power attenuation coefficient, a calculated parameter often given in fiber specifications, is a measurement of attenuation per unit length and is conventionally defined as $2 \alpha$. It differs from "loss" in having a base in the natural logs, rather than in logs of base 10 . Assuming $P_{0}$ is the total power input at the fiber end $(x=0)$, then at any point $x$ along a uniform fiber, the power $P(x)$ is

$$
P(x)=P_{0} \exp (-2 \alpha x)
$$

for single-mode fibers. Multimode fibers in which there is no mode coupling (equalization of speed of propagation of various modes caused by geometric imperfections or refractive index fluctuation) have a different attenuation coefficient for each mode. Since wavelength-dependent attenuation at the crystal boundaries of the polycrystalline structure of the KRS-5 fiber should cause considerable mode coupling, only one attenuation coefficient is reported in the experimental results that follow. 


\section{ATTENUATION MEASUREMENT RESULTS}

\section{Experimental Configurations}

The attenuation measurements made by Goebel of Ames Research Center in 1982 used the setup illustrated in figure 11. In this arrangement, an InSb photodiode detector was used (calibrated by the manufacturer to within $\pm 50 \%$ ) to measure power transmitted through a series of KRS-5 lenses. The lens in position $B$ could be replaced with a fiber and the two measurements compared. Wavelength resolution was achieved by the insertion of a circular variable filter in the optical path.

The configuration for the 1985 measurements was changed by replacing the focusing-lens system with a pinhole slightly larger than the fiber diameter on either end of the fiber (fig. 12). Transmission through the fiber was compared with transmission with the flber removed and replaced by a thin KRS-5 window to account for end losses. Better noise reduction was achieved by using a chopper in conjunction with a lock-in amplifier. The PbSnTe detector used was calibrated to within $\pm 1 \%$.

\section{Problems of Mechanical Instability}

Scattering at the fiber face would have been significantly reduced if we had been able to polish the fiber end. However, attempts to polish the fiber end were frustrated by it continually breaking. The large size of the individual crystals in the polycrystalline matrix was part of the problem, but recent research has shown that early-generation KRS-5 fibers suffer a deterloration of mechanical stability with age (ref. 15). This deterioration may be caused by residual strain from the fiber extrusion, causing separation at grain boundaries over time.

No evidence of grain separation was visible under high magnification from the outside surface of the broken end of the fiber. The vertical crack visible in the electron microscope photograph of figure 10 was probably caused by transverse stress on the fiber during extrusion. Its soft edges are indications that it is unlikely that it occurred later. The age-induced separation, if any, may not be visible from the exterior.

Investigation of this unexpected stability problem is just beginning, and better manufacturing techniques may save other researchers the difficulties encountered here.

\section{Results}

Figure 13 shows the results of these two series of measurements. The curve in the upper left at 1.2 to $3.4 \mu \mathrm{m}$ represents the 1982 data. Its shape may have been somewhat affected by the wavelength dependence of the focus of the lens used for comparison with the fiber. Measurements from 3 to $11 \mu \mathrm{m}$ were taken in 1985 . Details of data and error analysis can be found in appendix A. Figure 14 
shows measurements taken by Hidaka et al. (ref. 16) at Electrochemical Laboratory in Japan. Measurements in figure 15 were done by Harrington and Sparks (ref. 11) at Hughes Research and Scientific Research Center, respectively. These data have been replotted on a uniform scale in figure 16 to be compared with our data.

The Hidaka et al. (ref. 16) measurements are of losses in bulk KRS-5 with transmission length of about $1 \mathrm{~cm}$. Their results, at wavelengths longer than those of our study, show peaks associated with the presence of impurities. Dr. Harrington's results include data from attenuation measurements of two fibers. The fiber designated in figure 15 as number 2 also shows the absorption bands associated with impurity contamination; he points out that the $6.1 \mu \mathrm{m}$ band is due to the presence of water. Fiber number 1 is seemingly the best of all those tested here. Its attenuation exhibits a slope of $\lambda^{-2}$, suggested to be as a result of scattering at long surface grooves, the depth of which are less than $\lambda$ and the length of which are much greater than $\lambda$.

Our data lie at higher levels of attenuation than Harrington's and include obvious impurity bands at the longer wavelengths. The 1985 data under $8 \mu \mathrm{m}$ exhibits considerable linearity. The slope is $\lambda^{-0.57 \pm 0.55}$. The attenuation may be an effect of fiber ageing, strain, or other structural imperfections not yet identıfied. While the theoretical attenuation minimum of KRS-5 is at $7 \mu \mathrm{m}$ ( $\mathrm{ref} .17$ ), it is masked by high levels of attenuation from extrinsic causes.

CONCLUDING REMARKS

The case for the use of longer wavelengths than are currently employed in communications applications includes a reduction in losses from Rayleigh and other scattering and the potential ability to fabricate larger single-mode fibers. There are additionally detector and imaging applications, radiometric detection, and power transmission at IR laser wavelengths which suggest development of appropriate fibers.

Our measurements of attenuation in the range of $0.1 \mathrm{~dB} / \mathrm{cm}$ for wavelengths approaching $10 \mu \mathrm{m}$ show KRS-5 to be a promising future fiber material, particularly if mechanical parameters such as flexibility and strength can be substantially improved. 
APPENDIX A

TABLE A-1.- ATTENUATION OF KRS-5 FIBERS AT 1.3 to $3.4 \mu \mathrm{m}$

\begin{tabular}{cccc}
\hline$\mu \mathrm{m}$ & Transmittance & Attenuation, $\mathrm{dB} / \mathrm{cm}$ & Error $^{\mathrm{a}}$ \\
\hline 1.33 & 0.003 & 0.720 & 0.076 \\
1.41 & 0.009 & 0.584 & 0.033 \\
1.47 & 0.012 & 0.553 & 0.031 \\
1.54 & 0.014 & 0.531 & 0.030 \\
1.61 & 0.018 & 0.504 & 0.028 \\
1.67 & 0.023 & 0.472 & 0.026 \\
1.73 & 0.027 & 0.454 & 0.025 \\
1.88 & 0.036 & 0.415 & 0.023 \\
2.03 & 0.044 & 0.391 & 0.022 \\
2.15 & 0.050 & 0.374 & 0.021 \\
2.32 & 0.060 & 0.352 & 0.020 \\
3.11 & 0.071 & 0.330 & 0.018 \\
3.37 & 0.071 & 0.330 & 0.018 \\
\hline
\end{tabular}

TABLE A-2.- ATTENUATION OF KRS-5 FIBERS AT 3 to $11 \mu \mathrm{m}$

\begin{tabular}{llll}
\hline & & & \\
$\mu \mathrm{m}$ & Transmittance & Attenuation, dB/cm & $\begin{array}{c}\text { Error } \\
\times 10^{-3}\end{array}$ \\
\hline 3 & 0.014 & 0.140 & 1.83 \\
3.5 & 0.014 & 0.140 & 1.83 \\
3.75 & 0.022 & 0.125 & 1.65 \\
4 & 0.020 & 0.128 & 1.68 \\
4.5 & 0.033 & 0.112 & 1.48 \\
5 & 0.040 & 0.105 & 1.38 \\
5.5 & 0.042 & 0.104 & 1.36 \\
6 & 0.045 & 0.101 & 1.33 \\
6.5 & 0.057 & 0.094 & 1.18 \\
7 & 0.062 & 0.091 & 1.20 \\
7.5 & 0.066 & 0.089 & 1.17 \\
8 & 0.087 & 0.080 & 1.05 \\
8.5 & 0.058 & 0.093 & 1.23 \\
9 & 0.054 & 0.096 & 1.25 \\
9.5 & 0.081 & 0.083 & 1.08 \\
10 & 0.087 & 0.080 & 1.05 \\
10.5 & 0.092 & 0.078 & 1.03 \\
11 & 0.023 & 0.123 & 1.61 \\
\hline
\end{tabular}


Footnote for Tables $\mathrm{A} 1$ and A2:

${ }_{\text {The primary calculable sources of error are }}$

1. Blackbody source fluctuations, calculated from manufacturer's specifications (ref. 18) at $\mathrm{T} \pm\left(0.05 \% \mathrm{~T}+0.5^{\circ} \mathrm{C}\right)$, are small enough to be neglected.

2. The circular-variable filter gives wavelength resolution to $\pm 1 \mathrm{~nm}$, an insignificant source of measurement error. Wavelength calibration was from a Xenon emission-line source, the line positions of which are known to five-place precision.

3. Manufacturer's calibration for the thermopile detector was $\pm 50 \%$ for the 1982 data. Detector error was less than 1\% for the 1985 data.

4. Fiber/lens optical length measurement errors were taken into conslderation.

Error represents the square root of the variance, calculated from summing the squares of the partial derivatives (with respect to errors in flux measurement and fiber-length measurement) of the attenuation equation.

Not taken into consideration here, for the 1982 data, are the differences in front-surface scattering between the lens and the fiber. The lens and fiber have surfaces of a different shape, and this difference, added to the impossibility of a perfectly equal alignment of the angle of the incoming radiation when one element is substituted for the other, will have caused unequal acceptance scattering losses between them. 
Physical Properties of KRS-5

KRS -5 is a polycrystalline substance of refractive index 2.37 at $10 \mu \mathrm{m}$. (Compare optical glass with a refractive index of 1.5 at visible wavelengths.) This unusually high value is an advantage in fiber applications because for the same diameter fiber as one of lower index, the fiber will capture more light. Its chemical composition is $\operatorname{Th}(\mathrm{Br}, \mathrm{I})$. It has the relatively low melting point of $414^{\circ} \mathrm{C}$ and is lethally toxic when vaporized. Even in its solid state it should not be handled. Though not strongly hygroscopic (solubility $5 \times 10^{-2} \mathrm{~g} / 100 \mathrm{~g} \mathrm{H} \mathrm{H}_{2}$ ), even small amounts in the human body cause serious neurological damage; it is chemically similar to rat poison.

The fiber's tensile strength of $5,000 \mathrm{lb} / \mathrm{in} .^{2}$ is only one-fortieth that of silica fiber. Material dispersion zero is at $6.5 \mu \mathrm{m}$ (ref. 17). Its appeal as an optical fiber lies in its low theoretical attenuation and particularly in the location of its IR edge quite far in the IR at around $40 \mu \mathrm{m}$. It is one of the few flber materials suitable for use at the $10.6 \mathrm{CO}_{2}$-laser wavelength.

The fiber is fabricated by melting the starting material in a nitrogen atmosphere, then casting and sealing into glass ampoules before being crystallized. The resulting ingot is machined to a fiber preform shape, then extruded under high pressure through a diamond wire die into final form (ref. 19). 
REFERENCES

1. Sakuragi, S.: Polycrystalline KRS-5 Infrared Eibers. Advances in Infrared Fibers for Power Transmission, S.P.I.E. Proc., vol. 320, p.2.

2. Gannon, J. R.: Materials for Mid-infrared Waveguides. Opt. Eng., vol. 23, no. 2, Mar./Apr. 1984, p. 217.

3. Videau, J. J.; Dubois, B.; and Portier, J.: Verres a Base de Fluoure d'Indium. Chimin de l'Etat Solide, C. R. Acad. Sc. Paris, vol. 297, Serie II, 17 Oct. 1983, p. 484.

4. Allan, W.: Fibre Optics, Theory and Practice. Plenum Press (London), 1973, p. 1.

5. Kapron, F. D.; Keck, D. B.; and Maurer, R. D.: Radiation Losses in Glass Optical Waveguides. Appl. Phys. Letters, vol. 17, 1970, pp. 423-425.

6. Kapany, N. S.: Fiber Optics. Academic Press, 1967.

7. Yashita, T. M.; and Manabe, T.: Infrared Optical Fibers. IEEE J. Quantum Electr., vol. QE-18, no. 10, Oct. 1982.

8. Born, Max; and Wolf, Emil: Principles of Optics. Pergamon Press (Oxford), 1980 , pp. 634-652, 664 .

9. Pinnow, D. A.; Rich, T. C.; Ostermayer, F. W. Jr.; and DiDomenico, M. Jr.: Fundamental Optical Attenuation Limits in the Liquid and Glassy state with Application to Fiber Optical Waveguide Materials. Appl. Phys. Letters, vol. 22, no. 10, 15 May 1973, pp. 527-529.

10. Sakuragi, S.; Imagawa, K.; Salto, M.; and Kotani, H.: IR Transmıssion Capabilities of Thallium Halide and Silver Hallde Optical Fibers. Opt. Letters, vol. 6, no. 631, Dec. 1981, pp. 93-94.

11. Harrington, J. A.; and Sparks, M.: Inverse-square Wavelength Dependence of Attenuation in Infrared Polycrystalline fibers. Opt. Letters, vol. 8, no. 4, Apr. 1983, pp. 223-225.

12. Hecht, Eugene; and Zajac, Alfred: Optics. Addison-Wesley Publishing Company (Reading, MA), 1979, p. 41.

13. Goodman, C. H. L.: Devices and Materials for 4 Micron Band Fibre-Optical Communication. IEE J., Solid State Electron. Devices, vol. 2, no. 5, Sept. 1978 , p. 131. 
14. Lines, M. E.: Scattering Losses in Optic Fiber Materials. I. A New Parameterization. J. Appl. Phys., vol. 11, 1 June 1984, p. 4052.

15. Harrington, J. A.: A New Frontier for Optical Fibers. Opt. Spectra, Feb. 1981, pp. 39-41.

16. Hidaka, T.; Morikawa, T.; and Shimada, J.: Spectroscopic Small Loss Measurements on Infrared Transparent Materials. Appl. Opt., vol. 19, 15 Nov. 1980 , p. 3764 .

17. Rodney, W. S.; and Malitson, I. H.: J. Opt. Soc. A., vol. 46 (1956), from Harshaw Chemical Company: Technical Brochure (Solon, OH), 1967, p. 59.

18. Infrared Industries, Santa Barbara, CA, IR-463 Blackbody Source Product Manual, 1982.

19. Yashıta, T. M.; and Manabe, T.: Infrared Optical Fibers. IEEE J. Quantum Electr., vol. QE-18, no. 10, Oct. 1982, p. 1441. 


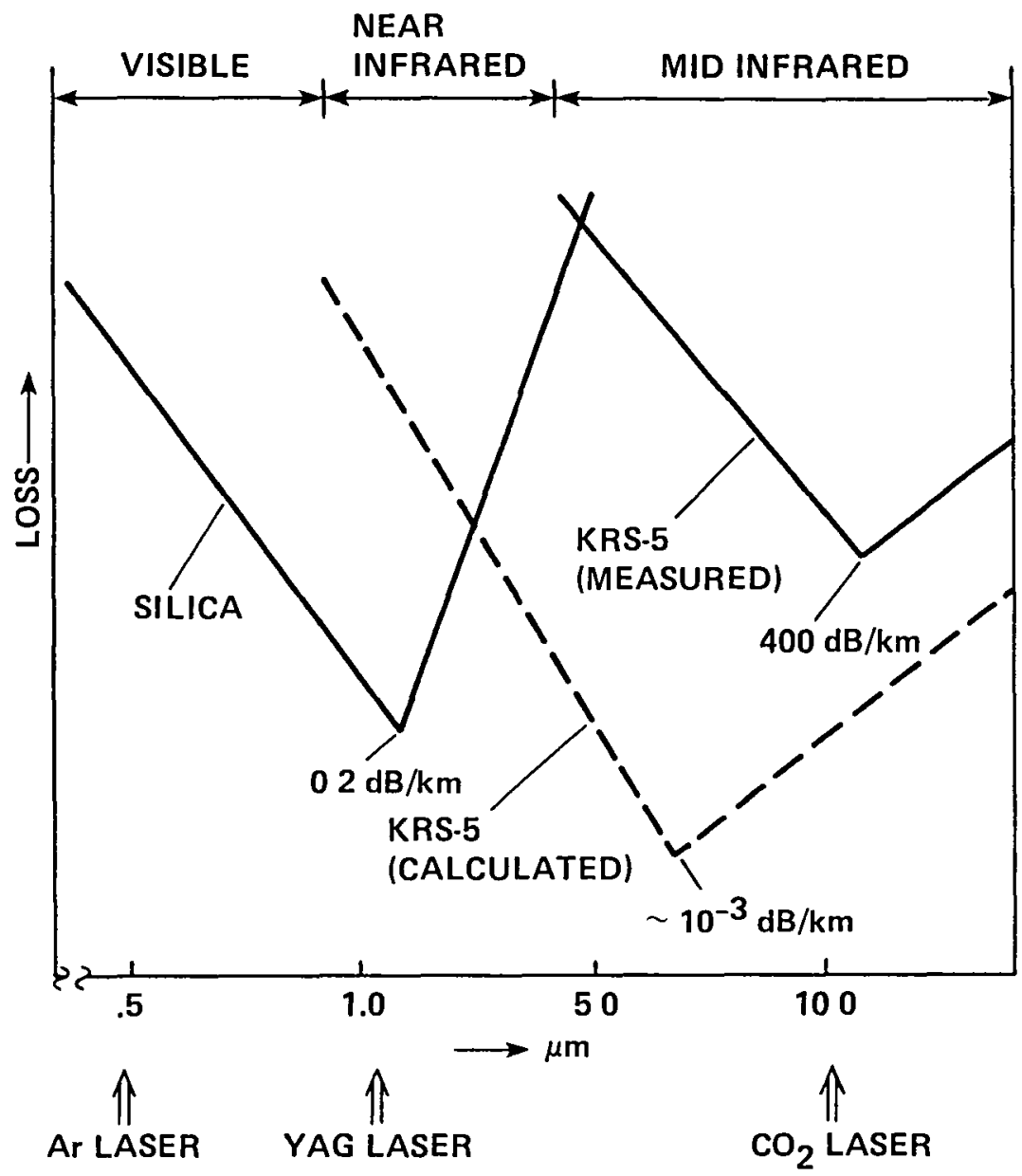

Figure 1.- Losses: Silica and KRS-5.

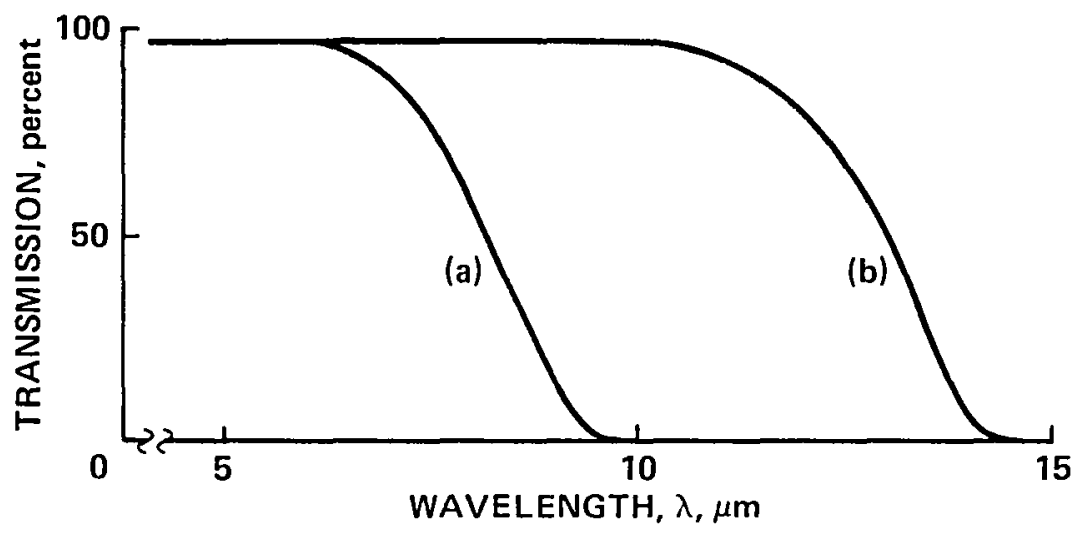

Figure 2.- Transmission: Indium fluoride glasses. 


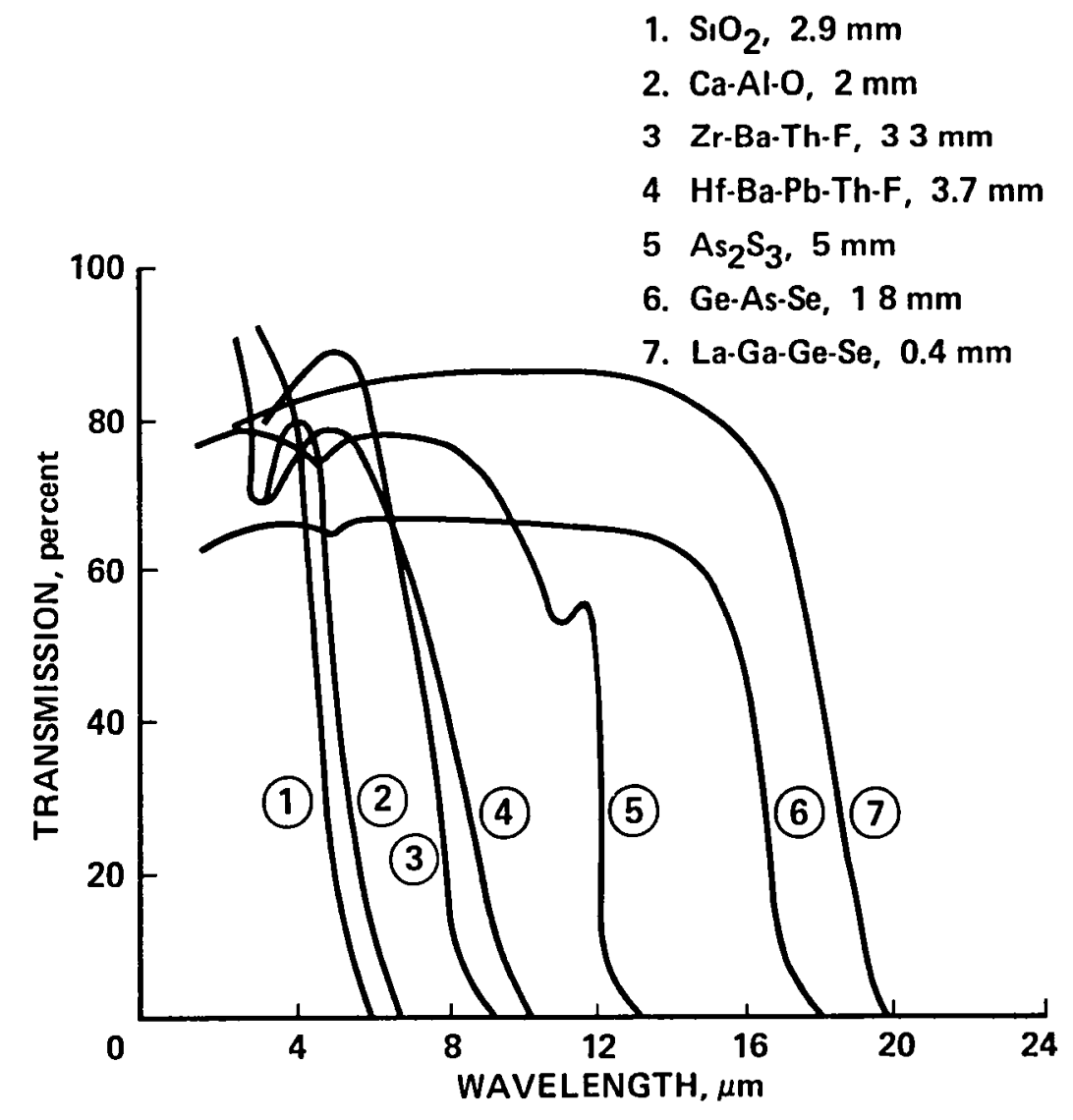

Figure 3.- Transmission of various glasses and crystals.

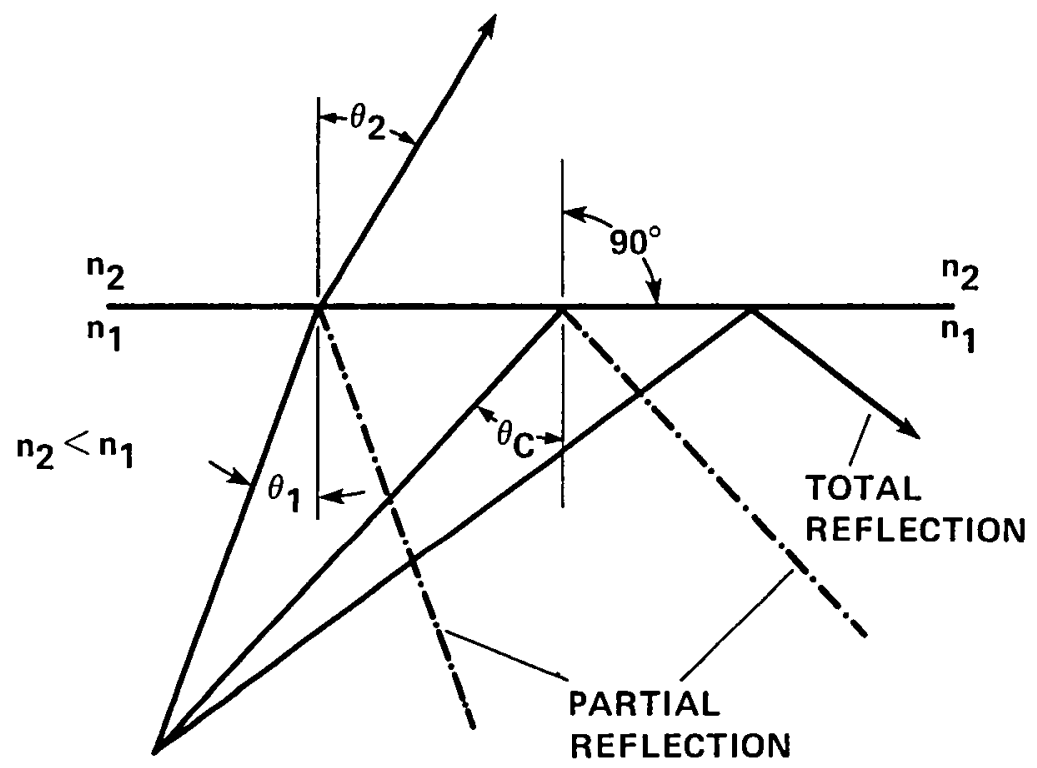

Figure 4.- Snell's Law and total internal reflection. 


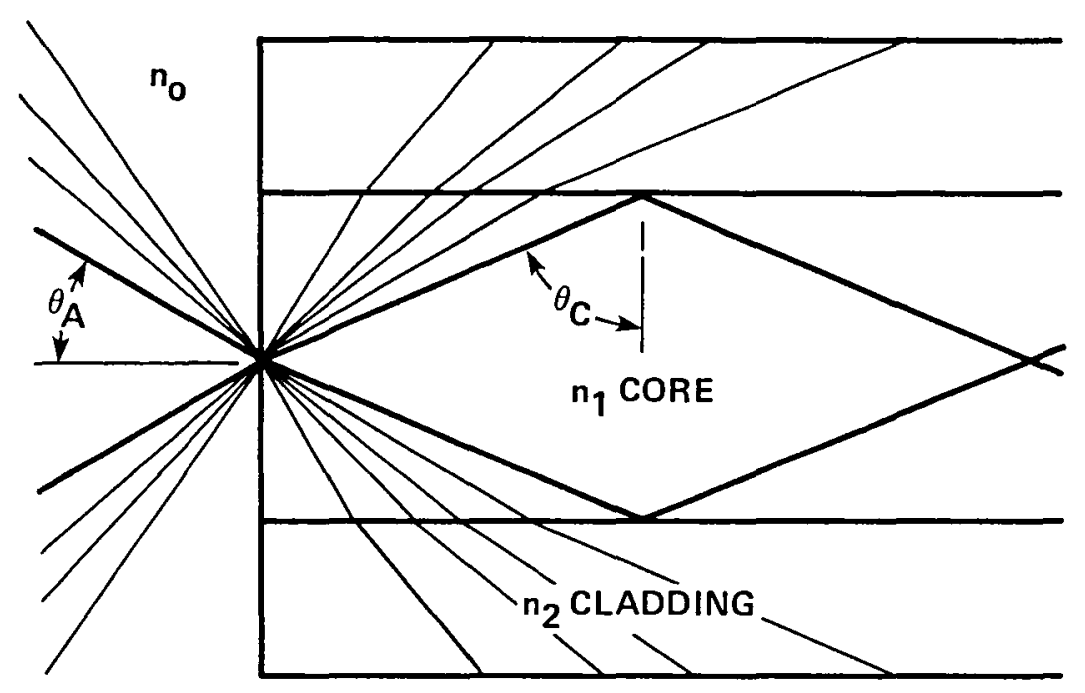

Figure 5.- Numerical aperture.

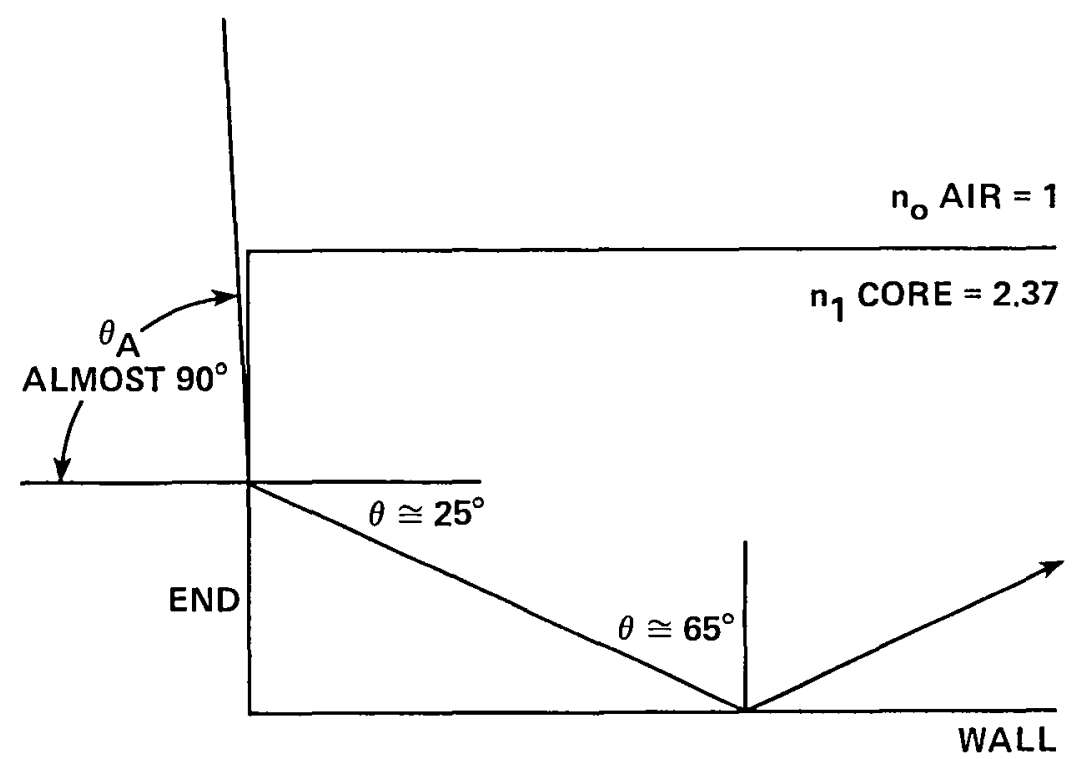

Figure 6.- Total internal reflection in a KRS-5 fiber. 


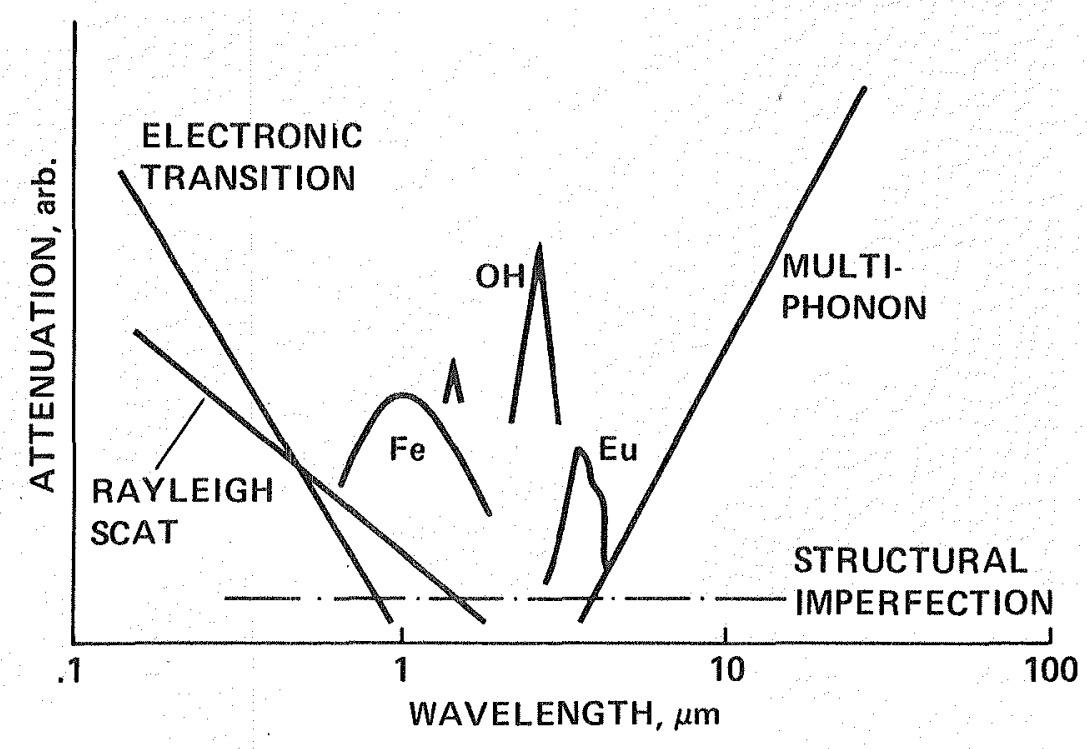

Figure 7.- Optical attenuation mechanisms in fibers.

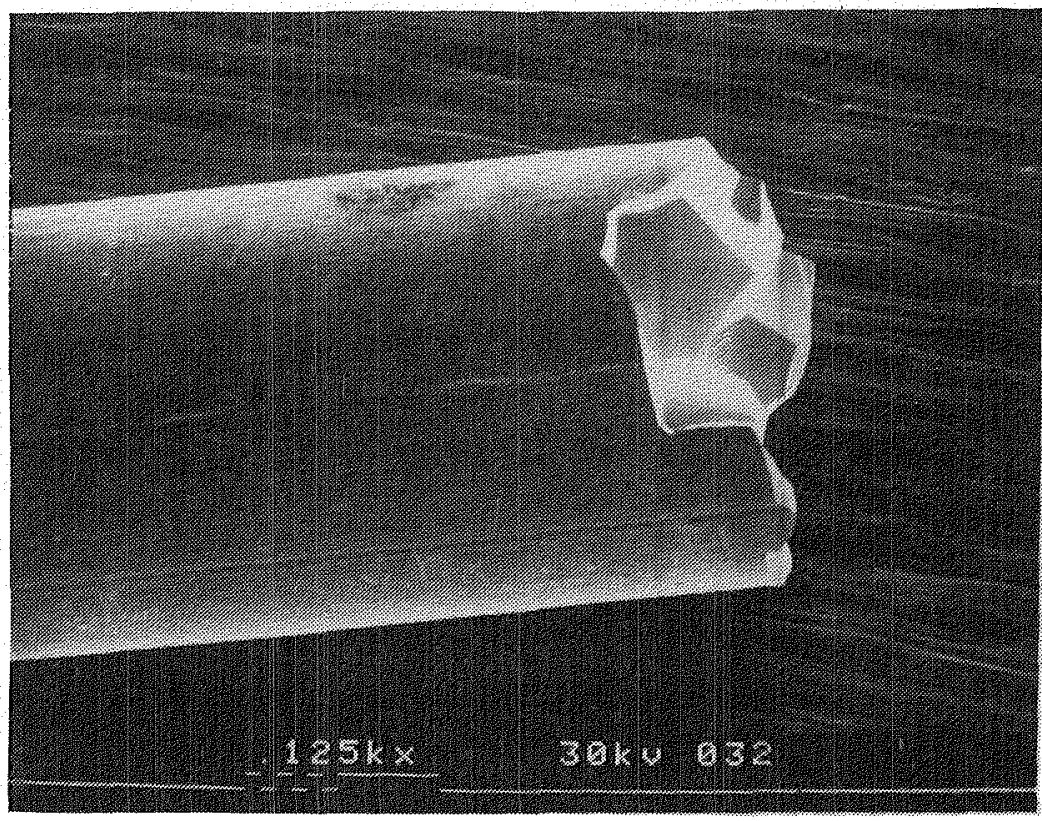

Figure 8.- KRS-5 fiber magnified 125 times, Harshaw manufacture. The length of the line under " $5 \mathrm{kx}$ " represents $100 \mu \mathrm{m}$. 


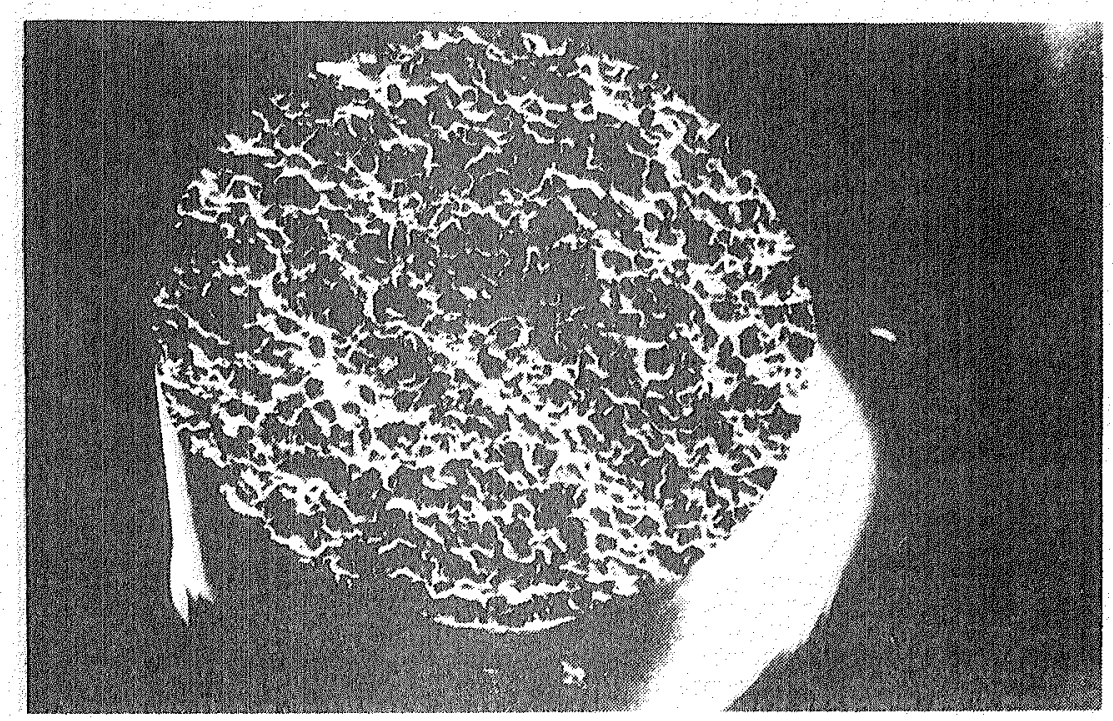

Figure 9.- KRS-5 fiber fabricated by Electrotechnical Laboratory, Ibaraki, Japan. Photo courtesy of the American Ceramic Society, Inc.

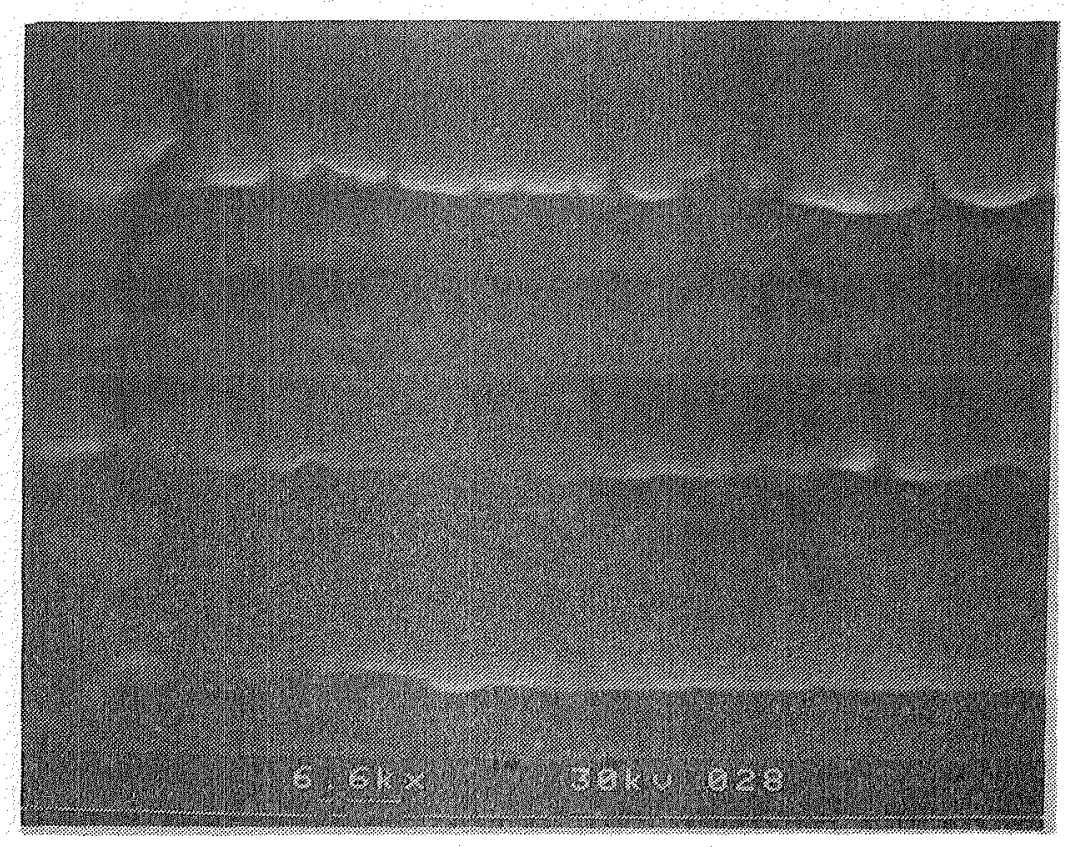

Figure 10.- KRS-5 fiber surface magnified 6600 times. The length of the line under "6k" represents $1 \mu \mathrm{m}$. 


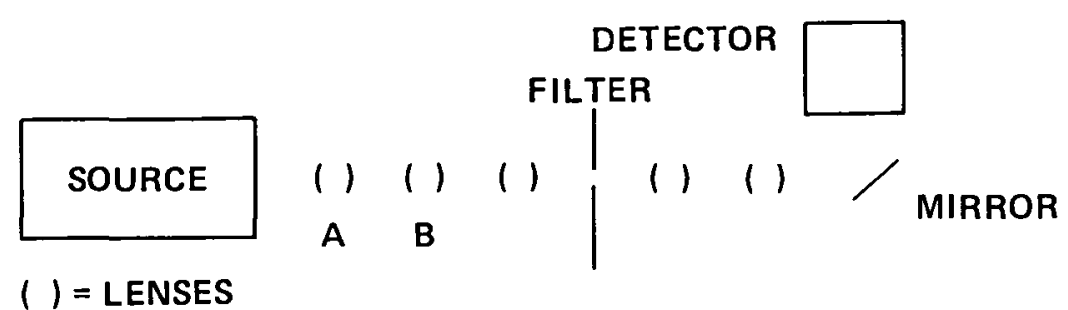

Figure 11.- Experimental configuration, 1982 data.

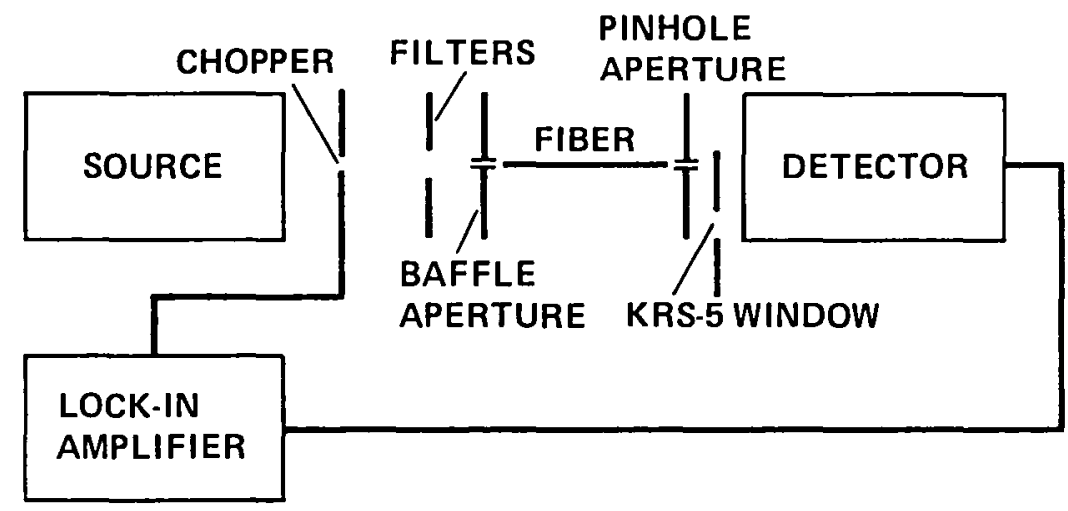

Figure 12.- Experimental configuration, 1985 data. 


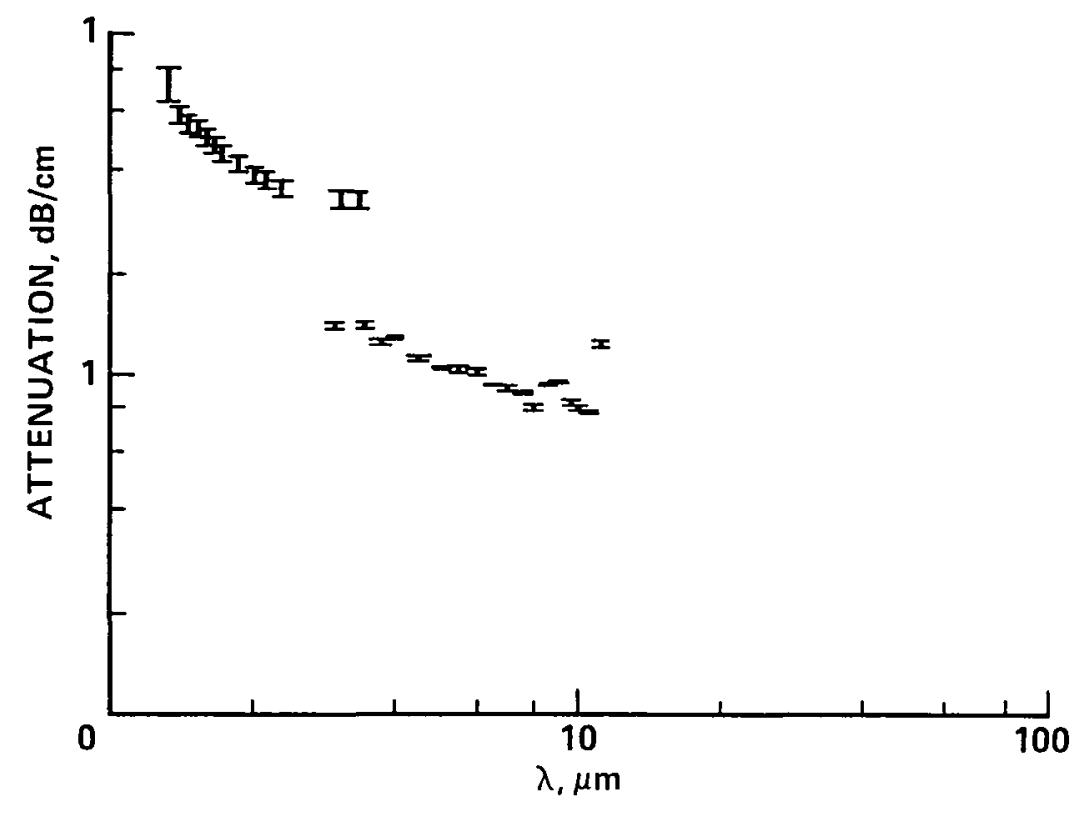

Figure 13.- Attenuation of KRS-5, Ames Research Center measurements.

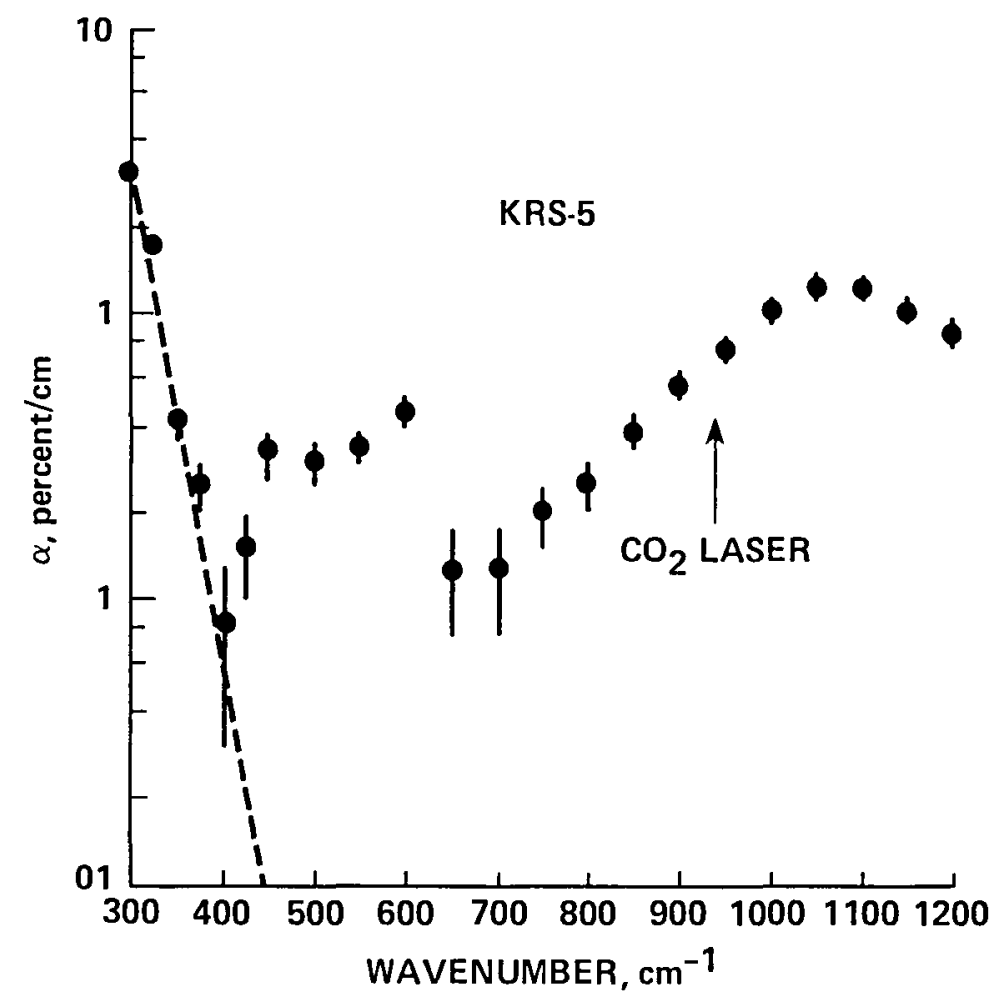

Figure 14.- Attenuation of KRS-5, Hidaka et al. 


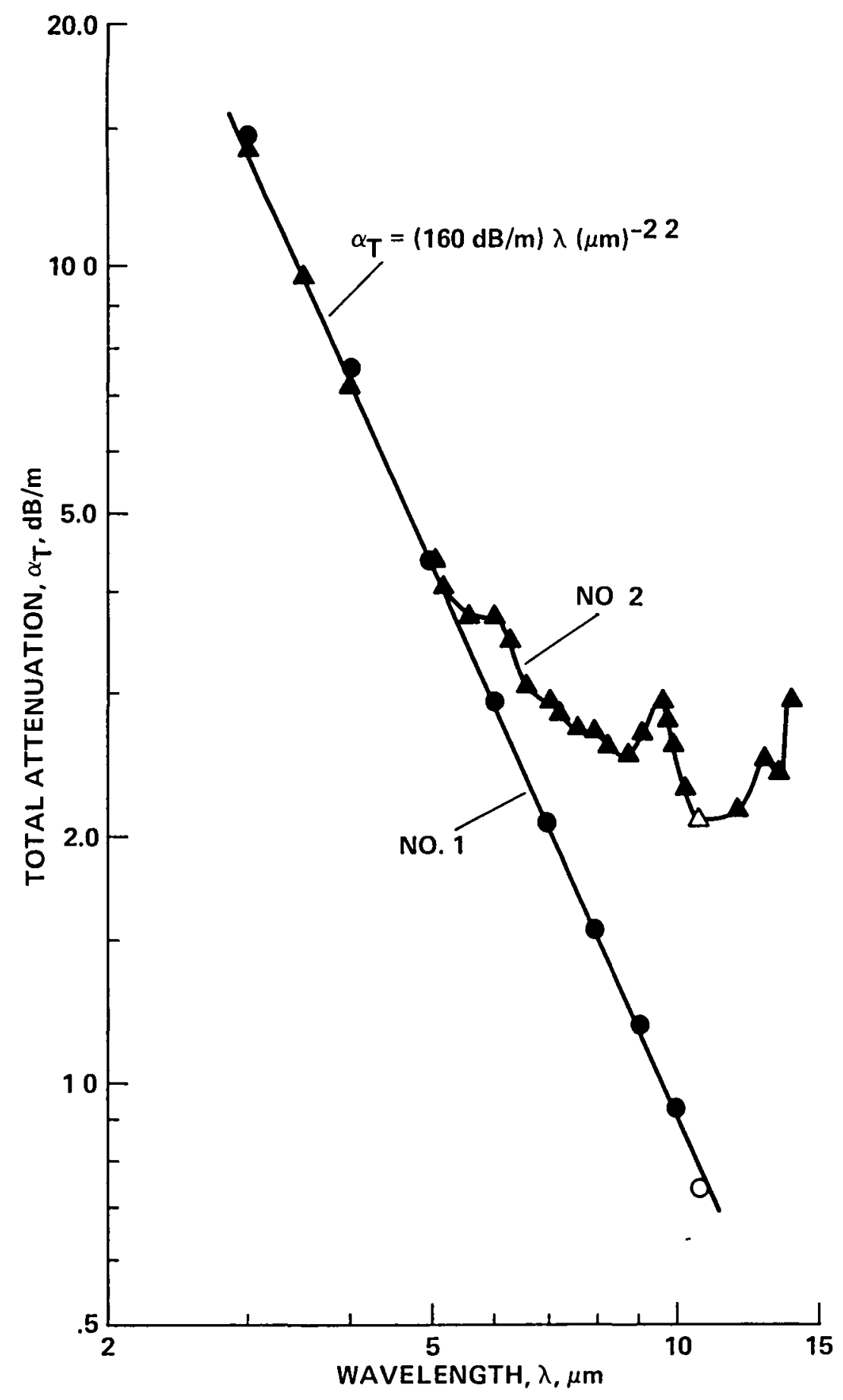

Figure 15.- Attenuation of KRS-5, Harrington. 


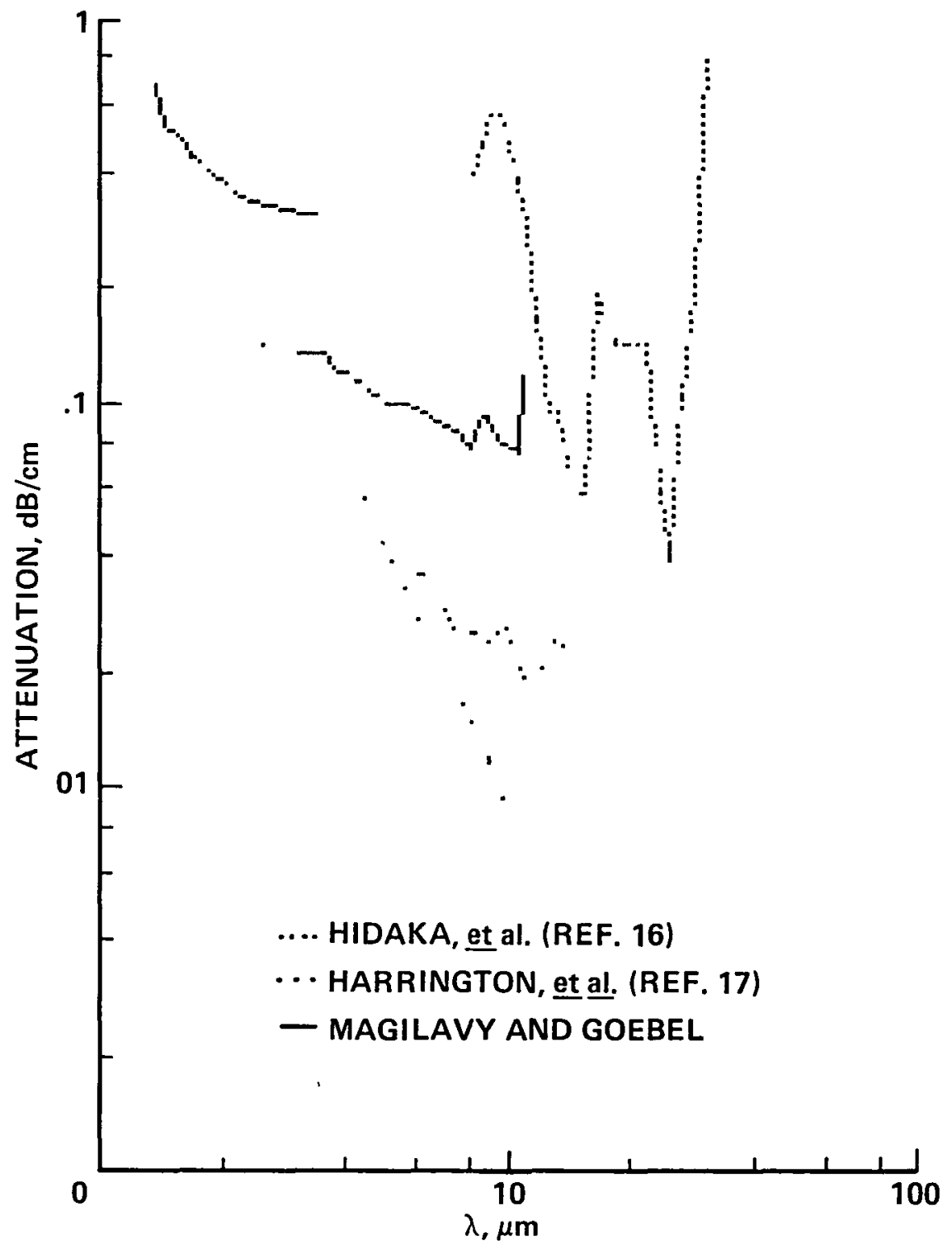

Figure 16.- Attenuation of KRS-5, comparison of research. 


\begin{tabular}{|c|c|c|c|c|}
\hline $\begin{array}{l}1 \text { Report No } \\
\text { NASA TM-86817 }\end{array}$ & \multicolumn{2}{|c|}{2 Government Accession No } & \multicolumn{2}{|c|}{3 Recipient's Catalog No } \\
\hline \multirow{2}{*}{\multicolumn{3}{|c|}{$\begin{array}{l}4 \text { Title and Subtıtle } \\
\text { INFRARED ATTENUATION OF THALLIUM BROMO-IODIDE FIBERS }\end{array}$}} & \multicolumn{2}{|c|}{$\begin{array}{l}5 \text { Report Date } \\
\text { February } 1986\end{array}$} \\
\hline & & & \multicolumn{2}{|c|}{6 Performing Organization Code } \\
\hline \multicolumn{3}{|c|}{$\begin{array}{l}7 \text { Author(s) } \\
\text { Bery1 Magilavy (Informatics, Inc., Palo Alto, CA) } \\
\text { and John Goebel }\end{array}$} & \multicolumn{2}{|c|}{$\begin{array}{l}8 \text { Performing Organization Report No } \\
\text { A-85370 }\end{array}$} \\
\hline \multirow{2}{*}{\multicolumn{3}{|c|}{$\begin{array}{l}9 \text { Performing Organization Name and Address } \\
\text { Ames Research Center } \\
\text { Moffett Field, CA } 94035\end{array}$}} & \multicolumn{2}{|l|}{10 Work Unit No } \\
\hline & & & \multicolumn{2}{|c|}{11 Contract or Grant No } \\
\hline \multirow{2}{*}{\multicolumn{3}{|c|}{$\begin{array}{l}12 \text { Sponsoring Agener Name and Address } \\
\text { National Aeronautics and Space Administration } \\
\text { Washington, DC } 20546\end{array}$}} & \multicolumn{2}{|c|}{$\begin{array}{l}\text { 13 Type of Report and Period Covered } \\
\text { Technical Memorandum }\end{array}$} \\
\hline & & & \multicolumn{2}{|c|}{$\begin{array}{l}14 \text { Sponsoring Agency Code } \\
\text { T-6986 }\end{array}$} \\
\hline \multicolumn{5}{|c|}{$\begin{array}{l}15 \text { Supplementary Notes } \\
\text { Point of contact: John Goebe1, Ames Research Center, MS 244-10, } \\
\text { Moffett Field, CA } 94035 \\
\text { (415) } 694-6525 \text { or FTS } 464-6525\end{array}$} \\
\hline \multicolumn{5}{|c|}{$\begin{array}{l}16 \text { Abstract } \\
\text { Analysis of attenuation measurements in the near infrared of an unclad } \\
\text { fiber of Thallium Bromo-Iodide (Th }(B R, I)) \text {, a polycrystalline thallium halide, } \\
\text { is presented. The first section of the paper is devoted to a general over- } \\
\text { view of the properties of fiber optics. Two groups of attenuation measure- } \\
\text { ments, for the region } 1.2 \text { to } 3.4 \mu \mathrm{m} \text { and for } 3 \text { to } 11 \mu \mathrm{m} \text {, respectively, are } \\
\text { presented, analyzed, and compared with those of two other groups of } \\
\text { researchers. Materials details of } \mathrm{Th}(\mathrm{Br}, \mathrm{I}) \text { are included as appendix A. }\end{array}$} \\
\hline \multicolumn{5}{|c|}{ 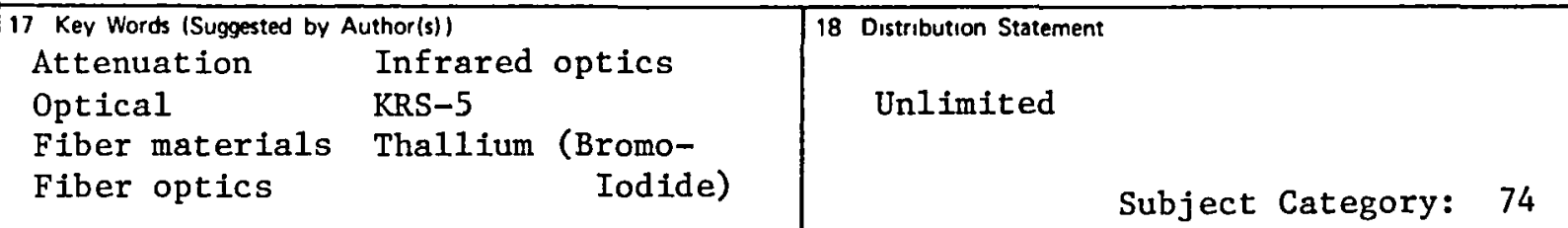 } \\
\hline $\begin{array}{r}19 \text { Security Cassif (of this repo } \\
\text { Unclassif ie }\end{array}$ & $\begin{array}{r}20 \text { Security Clas } \\
\text { Un }\end{array}$ & $\begin{array}{l}\text { of this page) } \\
\text { ssified }\end{array}$ & $\begin{array}{c}21 \text { No of Pages } \\
27\end{array}$ & $22 \begin{array}{l}\text { Price } \\
\mathrm{AO} 2\end{array}$ \\
\hline
\end{tabular}

-For sale by the National Technical Information Service, Springfield, Virginia 22161 


\section{End of Document}

